\title{
Multicriteria plan optimization in the hands of physicians: a pilot study in prostate cancer and brain tumors
}

Birgit S. Müller ${ }^{1,2,3^{*}}$, Helen A. Shih', Jason A. Efstathiou', Thomas Bortfeld ${ }^{1}$ and David Craft ${ }^{1}$

\begin{abstract}
Background: The purpose of this study was to demonstrate the feasibility of physician driven planning in intensity modulated radiotherapy (IMRT) with a multicriteria optimization (MCO) treatment planning system and template based plan optimization. Exploiting the full planning potential of MCO navigation, this alternative planning approach intends to improve planning efficiency and individual plan quality.

Methods: Planning was retrospectively performed on 12 brain tumor and 10 post-prostatectomy prostate patients previously treated with MCO-IMRT. For each patient, physicians were provided with a template-based generated Pareto surface of optimal plans to navigate, using the beam angles from the original clinical plans. We compared physician generated plans to clinically delivered plans (created by dosimetrists) in terms of dosimetric differences, physician preferences and planning times.

Results: Plan qualities were similar, however physician generated and clinical plans differed in the prioritization of clinical goals. Physician derived prostate plans showed significantly better sparing of the high dose rectum and bladder regions ( $p(D 1)<0.05$; $D 1$ : dose received by $1 \%$ of the corresponding structure). Physicians' brain tumor plans indicated higher doses for targets and brainstem ( $p(D 1)<0.05)$. Within blinded plan comparisons physicians preferred the clinical plans more often (brain: 6:3 out of 12, prostate: 2:6 out of 10) (not statistically significant). While times of physician involvement were comparable for prostate planning, the new workflow reduced the average involved time for brain cases by 30\%. Planner times were reduced for all cases. Subjective benefits, such as a better understanding of planning situations, were observed by clinicians through the insight into plan optimization and experiencing dosimetric trade-offs.

Conclusions: We introduce physician driven planning with MCO for brain and prostate tumors as a feasible planning workflow. The proposed approach standardizes the planning process by utilizing site specific templates and integrates physicians more tightly into treatment planning. Physicians' navigated plan qualities were comparable to the clinical plans. Given the reduction of planning time of the planner and the equal or lower planning time of physicians, this approach has the potential to improve departmental efficiencies.
\end{abstract}

Keywords: IMRT, Multicriteria optimization, Efficiency, Physician IMRT planning

\footnotetext{
* Correspondence: birgit.mueller@tum.de

'Department of Radiation Oncology, Massachusetts General Hospital, Harvard

Medical School, Boston, MA, USA

${ }^{2}$ Department of Radiation Oncology, Klinikum rechts der Isar, Technical

University of Munich, Ismaninger Straße 22, 81675 Munich, Germany

Full list of author information is available at the end of the article
} 


\section{Background}

The creation of a radiotherapy treatment plan is a stepwise process involving a diverse mix of staff. In clinical practice treatment plans are usually generated by dosimetrists or medical physicists ${ }^{1}$, translating written clinical prescriptions into dose distributions. The treatment plan optimization is a multicriterial problem which leads to inevitable trade-offs between targets and organs at risk (OAR) such that not all clinical goals can always be fulfilled [1-3]. Potential dosimetric conflicts might not be obvious before the initiation of planning and thus might not be addressed in the prescription. Clinical decisions have to be made during planning as they arise. Often planners ${ }^{1}$ spend much time trying to find a compromise between the different clinical goals, which might not be the trade-offs most preferred by the physician. When plans are presented to the physicians for clinical approval, it is often the first time the physician has the opportunity to review the dose distribution and many intermediate decision points with minimal or no physician input may have been made.

Many physicians accept a plan if it fulfills the prescribed dosimetric goals but it may not represent the most suitable compromise between different objectives. Being aware of the possibilities and weighing associated clinical consequences physicians might select a different trade-off. Making those clinical trade-off decisions should ultimately be in the hands of the staff trained to make them, i.e. the physicians.

Moreover, this current clinical planning practice frequently results in iterative plan adaptations until the plan is approved by the physician - a time consuming process for both physician and planner.

In addition to the interaction between physicians and planners for clinical decisions, the actual planning is frequently inefficient. Planning is often a trial-and-error process, with the quality of the final plan dependent on the skills or personal perception of the planner. Planners use different helper structures and parameters based on experience and knowledge. Many attempts to standardize treatment planning and improve its consistency by finding class solutions and using knowledge based planning have been reported [4-9].

Multicriteria optimization (MCO) has proven to be an efficient treatment planning method, both in terms of planning time and dosimetric quality $[1,10-12]$. It is applicable to problems where there is no single clear optimal solution, but instead the problem requires compromises. These compromises are described mathematically on the so-called Pareto surface of optimal plans $[13,14]$. Plans on the surface cannot be improved in one criterion without worsening another $[13,15]$. MCO planning software eliminates the time-consuming trial-and-error process of selecting suitable weighting factors in conventional IMRT planning [16-19] and visualizes these dosimetric tradeoffs. It provides a tool to interactively navigate on the surface and investigate the trade-offs $[16,17]$ which makes planning more intuitive but yet may not be fully exploited in the current clinical planning routine.

One approach to make use of the MCO system, and to improve treatment planning, both in achieving the desired dosimetric goals and in increasing efficiency, is to involve the physician at an earlier stage of the planning procedure.

We suggest physician driven planning as an alternative planning procedure, which - similar to the current workflow - consists of a collaboration of physicians and planners, but differs in the order and responsibility of involved tasks. Physician driven planning utilizing MCO treatment planning software avoids the "human iteration loop" between physicians and planners by providing physicians the control over trade-offs, and allows them to tailor the treatment plans to the individual patient.

In a retrospective planning study, we demonstrate the feasibility of physician driven planning by template based optimization and physician plan navigation as a suitable planning procedure, and assess whether it has the potential to improve planning efficiency and quality. We compare clinically delivered plans, created by dosimetrists, to plans that physicians interactively navigated (Fig. 1).

\section{Methods}

The study is based on data from 12 brain tumor and 10 prostate cancer patients previously treated with MCO optimized intensity modulated radiotherapy (IMRT) (step and shoot) at Massachusetts General Hospital (MGH) and selected randomly out of the clinical database.

\section{Structure definition \\ Brain tumors}

The patients featured a variety of diagnoses and intracranial anatomical sites. The gross tumor volume (GTV) was defined based on integrated $\mathrm{CT}$ and magnetic resonance imaging from preoperative and postoperative studies. Clinical target volumes (CTV) were created to encompass additional regions of potential microscopic involvement. Prescriptions to the planning target volume (PTV) (CTV $+3 \mathrm{~mm})$ ranged from $36 \mathrm{~Gy}(12 \times 3 \mathrm{~Gy})$ to 60 Gy $(30 \times$ 2 Gy). Standard OARs defined for all cases included the brainstem, chiasm, optic nerves, eyes, lenses, lacrimal glands, and cochleae. Tolerance doses varied dependent on the individual case, i.e. tumor location and prescribed dose. For 60 Gy commonly given constraints were amongst others: maximum dose $\mathrm{D}_{\max }$ (optic nerves and chiasm) <54 Gy, $\mathrm{D}_{\max }$ (orbits) < 45 Gy, $\mathrm{D}_{\max }$ (brainstem) $<54$ Gy in center, $<60$ Gy on the surface, and a maximum of 57 Gy to $5 \%$ of the volume. 


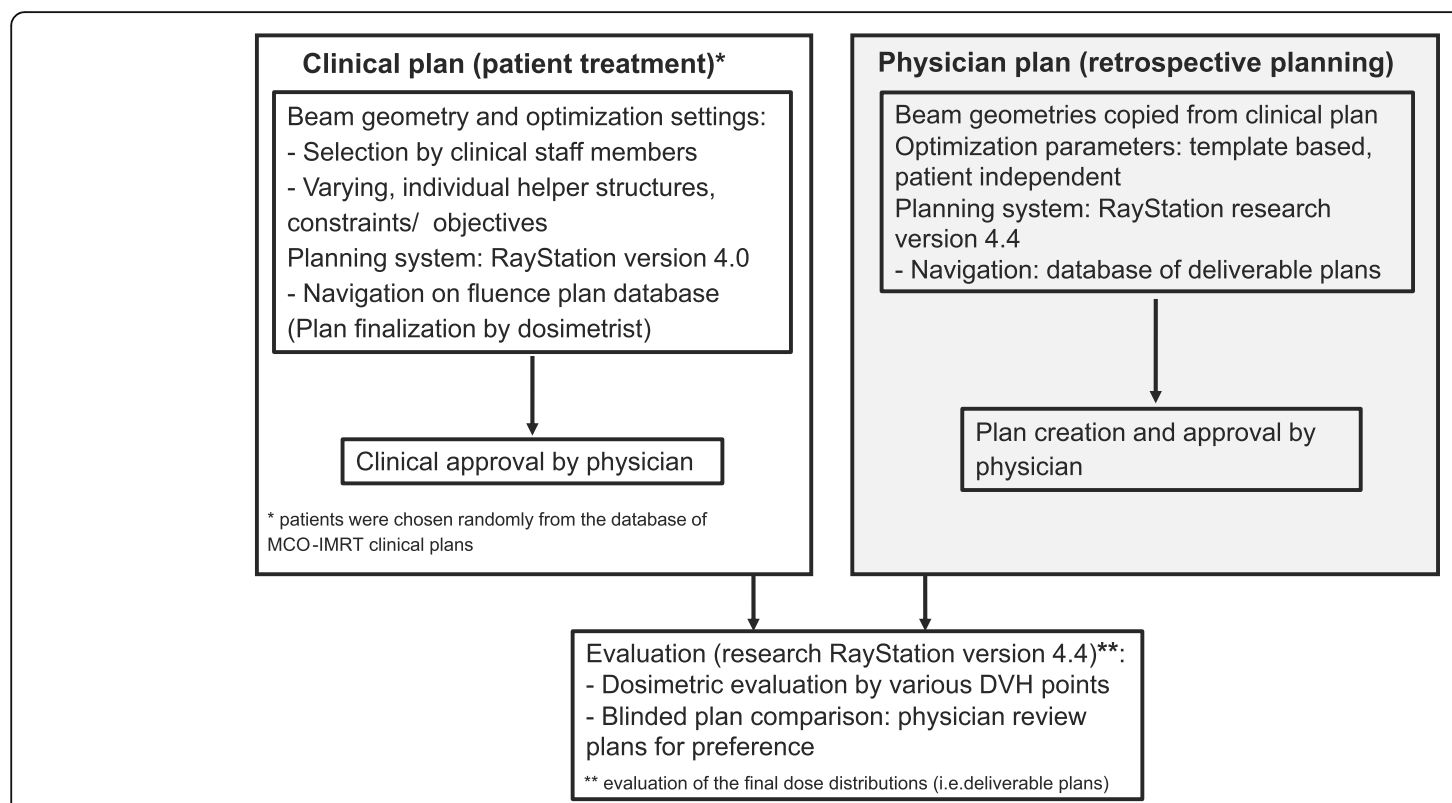

Fig. 1 Planning study workflow

\section{Prostate cancer}

All cases had undergone radical prostatectomy and were receiving postoperative radiation therapy. The CTV (prostatic fossa), PTV, rectum, bladder (excluding (TV) and femoral heads were contoured for all patients. Small bowel, sigmoid, residual seminal vessels and penile bulb were added dependent on individual patients' anatomy and clinical situation. The prescribed dose to the PTV (CTV $+8 \mathrm{~mm}$, posterior: + $4 \mathrm{~mm})$ was $66.6 \mathrm{~Gy}(37 \times 1.8 \mathrm{~Gy})$. For other planning parameters see Table 1 .

\section{MCO treatment planning}

MCO-planning comprises the Pareto front calculation, the subsequent plan navigation and deliverable plan creation. The Pareto front is calculated by optimizing various weighted sums of prior defined treatment objectives. For $\mathrm{n}$ objectives at least $\mathrm{n}+1$ plans are calculated [15], and the maximum plan number is given by the software with approximately $4 \mathrm{n}$ (default usage for the study). Each treatment objective is represented in the treatment planning system (TPS) navigation interface by a slider (Fig. 2). It improves the corresponding objective function and updates the dose distribution in real time when moved by interpolating between pre-computed plans. After navigation, plans are finalized by multileaf collimator (MLC) sequencing and final dose calculation.

Clinical plans were generated in the commercial clinically implemented MCO-planning system RayStation (version 4.0; RaySearch, Stockholm, Sweden); retrospective physician driven plans were created in a research software version (research version 4.4) (Fig. 1). Whereas plan navigation in the commercial software is based on fluence maps, the research module allows for navigation on segmented plans which decreases dose differences of the final planning step [20,21] and may therefore be more suitable for physician driven planning. With regards to all other planning and dosimetric parameters, including the dose calculation, the planning systems are identical.

Table 1 Typical MCO-problem formulations for prostate (prescription: 66.6 Gy); EUD: equivalent uniform dose [29], * excluding PTV wall

\begin{tabular}{|c|c|c|c|}
\hline \multicolumn{2}{|l|}{ Constraints } & \multicolumn{2}{|l|}{ Objectives (slider) } \\
\hline \multirow[t]{2}{*}{ PTV } & Min $/$ max dose $=$ prescribed dose $+/-15 \%$ & PTV & 1) Min dose $=$ prescribed dose \\
\hline & & & 2) Max dose $=$ prescribed dose \\
\hline CTV & Min DVH: prescribed dose to $95 \%$ volume & CTV & Min dose $=$ prescribed dose \\
\hline PTV wall (1 cm ring around PTV) & Max dose $=$ prescribed dose $+3 \%$ & & \\
\hline Rectum/ bladder (excluding CTV) & Max dose $=$ prescribed dose $+5 \%$ & $\begin{array}{l}\text { Rectum/ bladder } \\
\text { (excluding CTV) }\end{array}$ & Dose-fall off: prescribed dose to $0 \mathrm{~Gy}$ in $1 \mathrm{~cm}$ \\
\hline \multirow[t]{2}{*}{ Normal tissue* } & Max dose $=$ prescribed dose & Normal tissue & Dose-fall off: prescribed dose to $0 \mathrm{~Gy}$ in $1 \mathrm{~cm}$ \\
\hline & & Femurs & EUD, $a=2$ \\
\hline
\end{tabular}




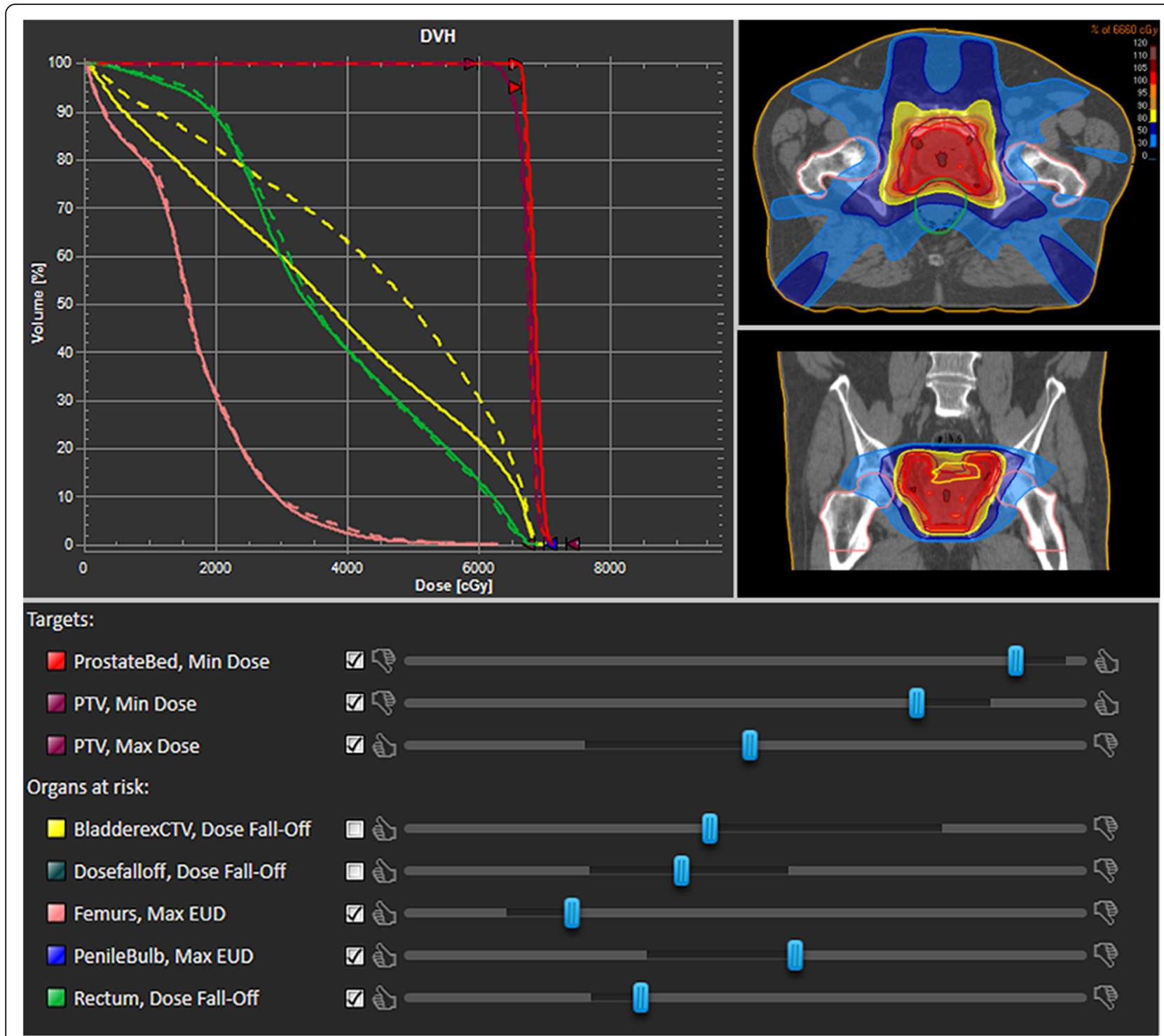

Fig. 2 Interface components of MCO-plan navigation: dose distributions are adjusted in real time by moving the sliders of different structures. The dosimetric changes are also visualized in the dose volume histogram: current (straight line) and previous (dashed line) dose

\section{Planning and optimization parameters}

The clinically chosen beam settings were also used for retrospective physician planning (prostate: 7 beams, brain tumors: 4-7 beams) to ensure that all found dose differences between the clinically and physician generated plans were the result of user preferences of plan nuances rather than fundamental factors such as beam direction. The clinical plans that served as our baseline comparison plans were made by the clinical dosimetry treatment planning staff who did not know that their plans would be used in a retrospective comparison study. At $\mathrm{MGH}$, treatment site specific optimization templates exist and are recommended for use. As planners often change these as they wish, clinically utilized planning helper structures and optimization parameters varied between planners and patients. For the retrospective planning study a database of Pareto optimal plans was created using mostly patient independent but site specific, self-developed, templates (Table 1) in order to test the idea that a template based Pareto-surface creation technique was suitable for physician-based MCO planning. The set of constraints contained loose minimum and maximum doses to prevent extreme under- and overdosage in the target and uncompromising doses to OARs, but not to restrain physicians in their options. A detailed explanation of the implemented objective functions can be found in [22].

\section{Physician plan navigation}

Physicians, who treated these patients before, were provided with the anonymized patients, the plan database 
and patient information (prescription, constraints and history); no access was given to the prior clinically treated plan. Prostate plan navigation (Fig. 2) was conducted with 9 sliders on average (max.10), brain tumor cases with 15 (max. 16), amongst those a maximum of three sliders for the target (two for the PTV, one for CTV or GTV) (Table 1).

\section{Plan evaluations and efficiency analysis}

The final clinical and physician dose distributions were analyzed by several dose volume histogram (DVH) values. Statistical evaluations were performed by paired t-tests and non-parametric signed rank tests. Two weeks after planning physicians were asked for their plan preference, for each patient case, in a blinded comparison. Preferences were rated as slightly or significantly different. The option "no preference" referred to equal plan quality.

We analyzed required planning times of planners and physicians for both procedures in a theoretical comparison of the main components, i.e. 1) beam selection (time $\left.t_{1}\right)$,2) selection plus creation of helper structures for the optimization (where applicable) and Pareto surface calculation $\left(t_{2}\right)$ and 3) navigation plus physician approval $\left(t_{3}\right)$.
Planners are involved in all steps $\left(t_{\text {planner }}=t_{1}+t_{2}+t_{3, p l}\right)$, physicians exclusively in the last step $\left(t_{\mathrm{ph}}=\mathrm{t}_{3, \mathrm{ph}}\right)$ (Fig. 3 ). Physicians' navigation times, including the deliverable plan generation, were recorded, and clinical times were determined based on departmental interviews of staff members. In clinical planning $(\mathrm{CP})$, the required times are driven by the number of iterations $\mathrm{N}$ between both staff members until a plan is approved by the physician. Average physician and planner times $\mathrm{t}_{3, \mathrm{ph}}(\mathrm{CP})$ and $\mathrm{t}_{3, \mathrm{pl}}(\mathrm{CP})$ were calculated by the time to coordinate between physician and planner, including the communication of new instructions, to review and approve the plan, multiplied by the number of iterations of plan adaptations before approval.

\section{Results}

\section{Dosimetric differences}

Most evaluated criteria did not show significant differences or trends but were spread around zero, $D_{\text {mean }}$ of the femoral heads (Fig. 4a) being a typical example. Prostate plans, generated by the physician, showed a better sparing of high dose regions of bladder and rectum, as demonstrated by the significant differences in V65(rectum) $(p=0.009)$ and V65(bladder) $(p=0.003)$ (relative volume receiving at least $65 \mathrm{~Gy}$ ). The PTV

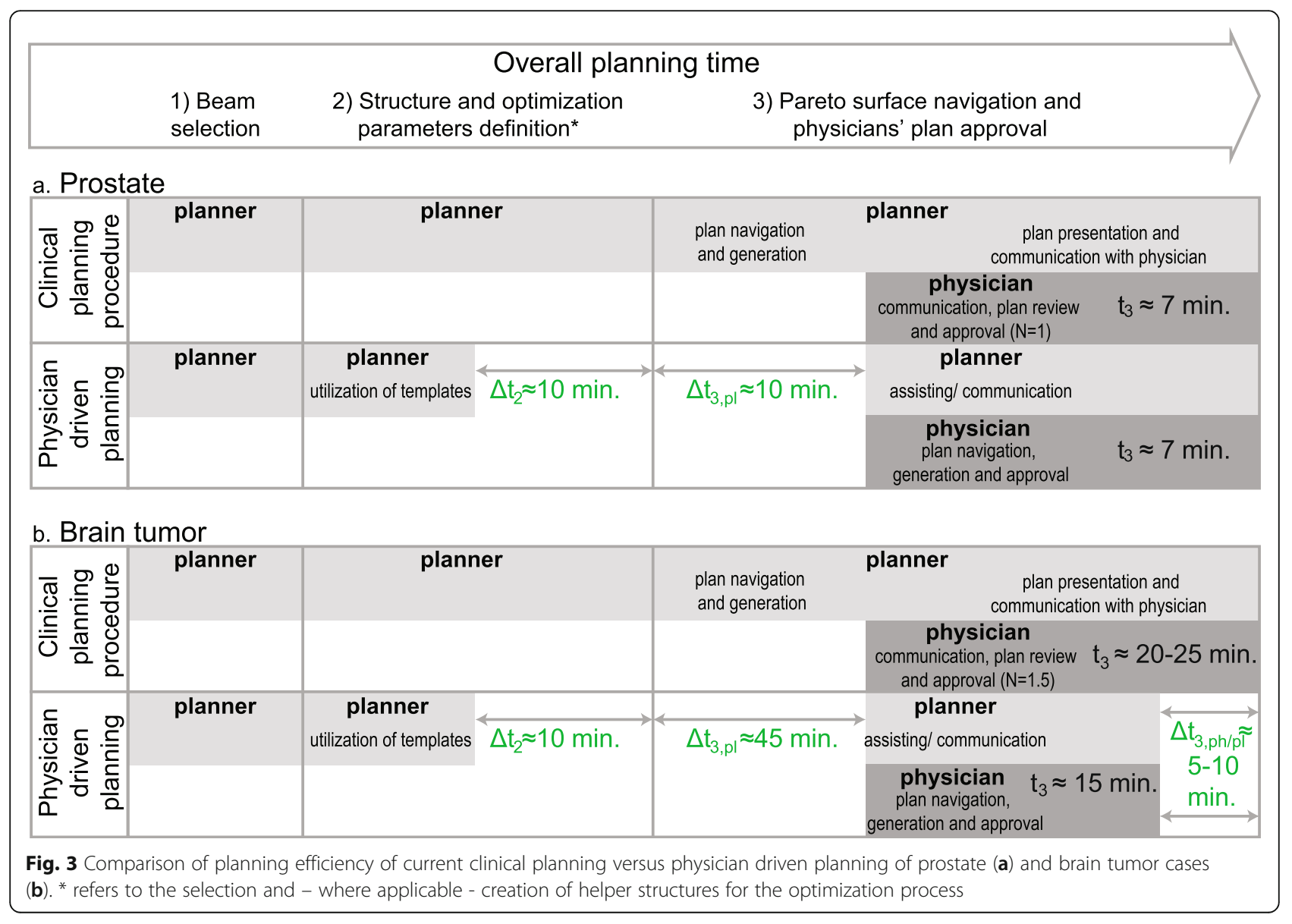



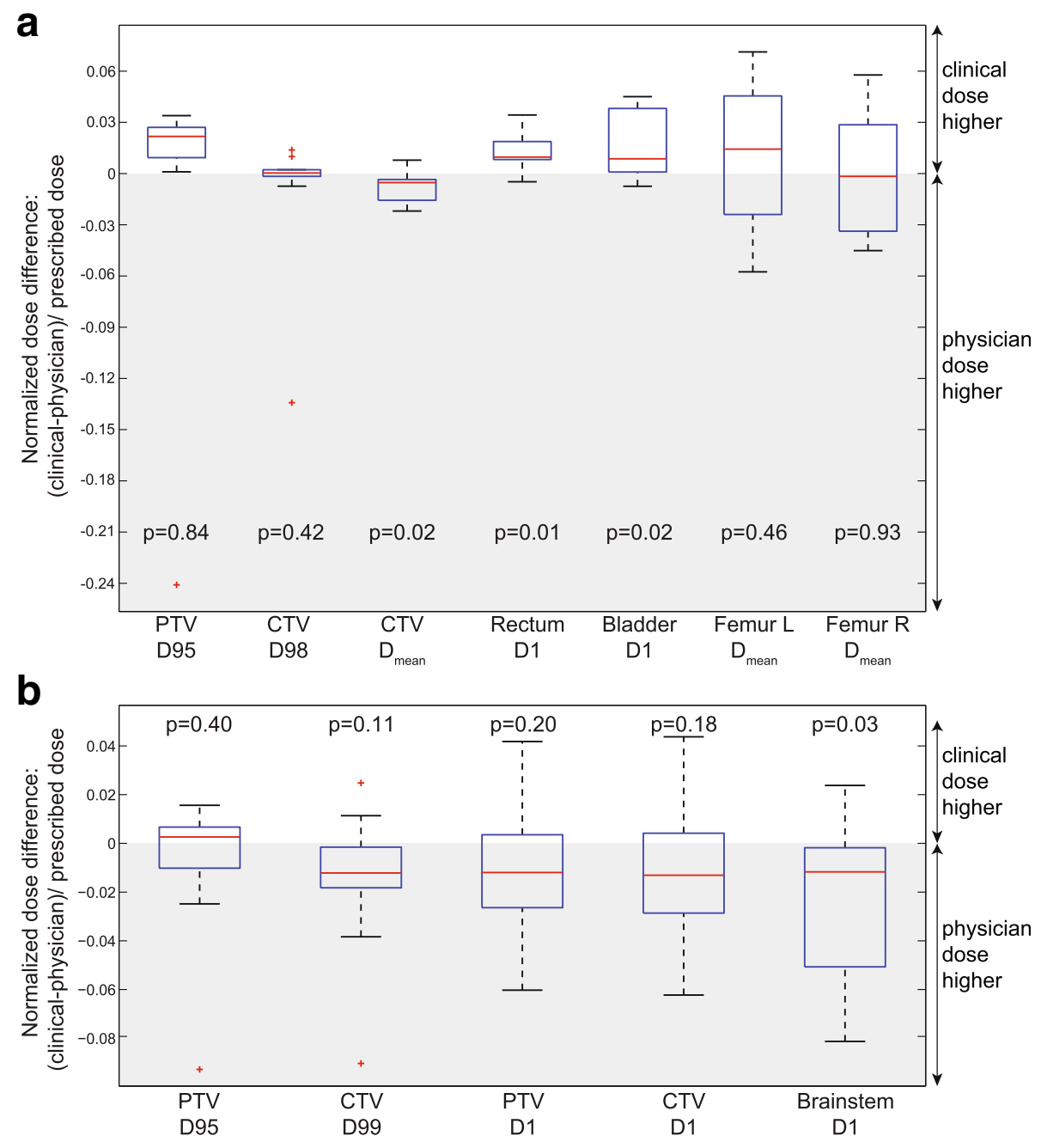

Fig. 4 a Normalized dose differences of prostate plans of performed t-tests; we also computed the non-parametric Wilcoxon signed rank pstatistics since some of the difference data do not pass the Lilliefors normality test (due to the outliers). Those $p$-values lead to the same conclusions as the p-values from the t-test. Starting with PTV D95, they are $0.08,0.70,0.03,0.004,0.04,0.43$, and 0.92. b Normalized dose differences of brain tumor plans of performed t-tests; p-values of the Wilcoxon signed rank test, starting at PTV D95, are 0.97, 0.05, 0.23, 0.20, 0.019; remarks: CTV refers to 11 CTVs and one GTV contour; brainstem statistics based on 11 contours

covered by the $98 \%$-isodose was significantly lower (V65 (PTV): $p=0.007)$ while the CTV received higher mean doses. The trend of greater PTV-coverage in clinical plans is presented in Fig. 4a by D95(PTV) (dose covering $95 \%$ of the PTV). One outlier did not follow this trend but showed a low D95(PTV) (due to small bowel sparing). Ignoring this plan in the statistical evaluations would lead to significance for D95(PTV) with $p<5 \%$ while the trend of all other criteria would be the same.

Physician derived brain tumor plans indicated a trend to higher doses in the targets and OARs, with a significantly higher maximum dose to the brainstem (D1(brainstem): $p=0.03$ ) (Fig. 4b). Dose distributions of one brain tumor and one prostate example showing clear dosimetric differences are shown in Fig. 5.
Trade-off plots of different dosimetric objectives present the chosen compromises by physicians and dosimetrists (Fig. 6). Except for the navigation to higher CTV doses for a cost in increased D1(brainstem) (Fig. 6b), no comprehensive trade-offs over all patients were found for brain tumor plans; chosen compromises differed between patients.

\section{Physicians' preferences}

The blinded plan comparison results of physicians' preference are presented in Table 2, which shows a slight preference for the clinically generated plans. The doctors decided all plans, whether selected or not, were clinically acceptable. However, for one brain tumor case (Fig. 5) the physician would have preferred to navigate again in 


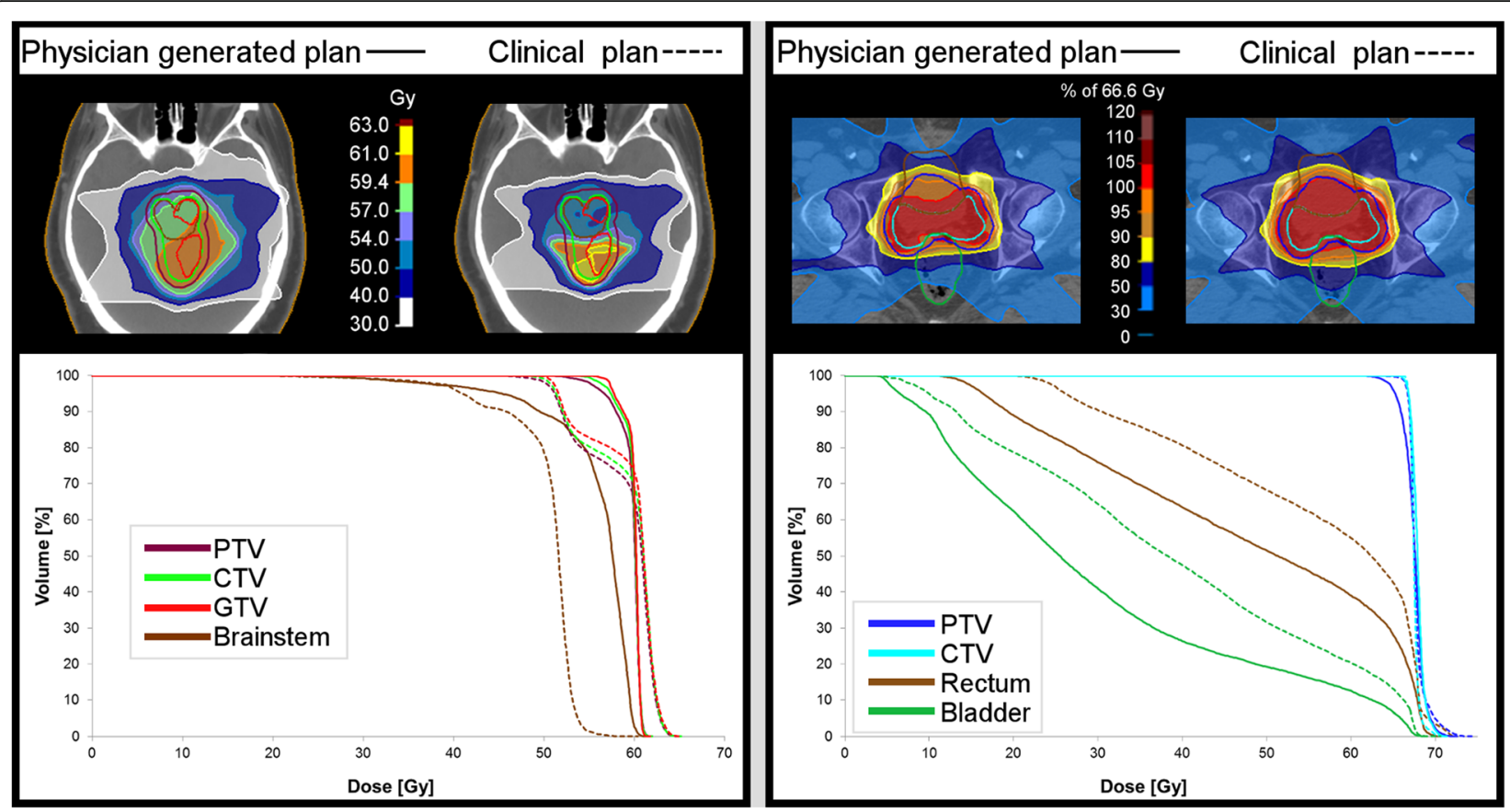

Fig. 5 Case examples of remarkable differences between clinical and physician planning for brain tumor and prostate; blinded plan comparison resulted in "no preference" for brain tumor (physician preferred an average of both) and in favor of physician prostate plan
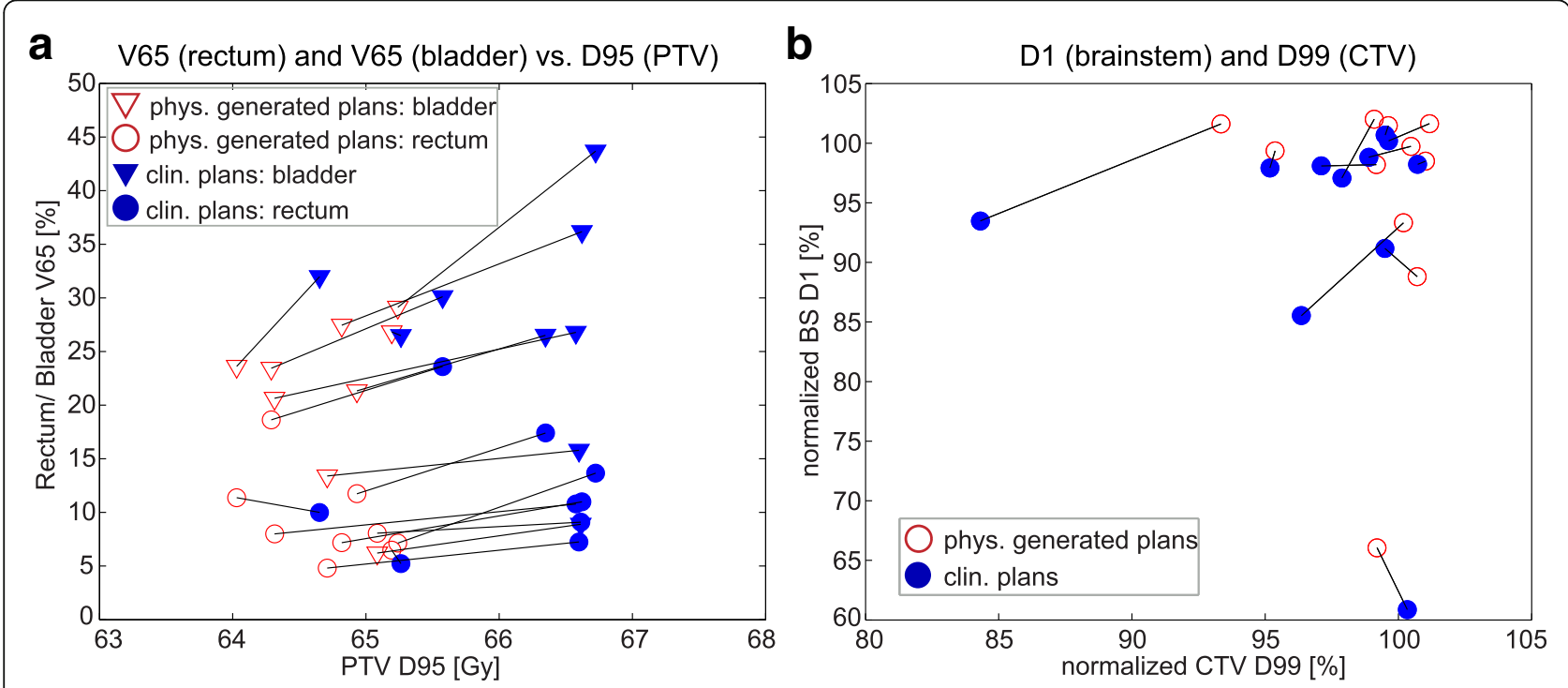

Fig. 6 Dosimetric trade-offs of selective DVH criteria for prostate (a) and brain tumor plans (b). Each data point represents the compromise between two dosimetric objectives of one plan; physician (phys.) generated plan results are linked to corresponding clinical (clin.) plan trade-offs. Nine prostate and 11 brain tumor cases are presented (excluding the prostate outlier, see Fig. 4a, and the brain case without brainstem contour). (note: plans which are superior in all presented DVH criteria than the corresponding plan were worse in at least one other dosimetric objective that is not included in the figure) 
Table 2 Physicians' plan preferences: results of blinded plan comparison; * physician $1=$ planning physician, physician $2=a$ non-planning involved physician

\begin{tabular}{|c|c|c|c|c|c|c|}
\hline & \multicolumn{2}{|c|}{ Brain tumors } & \multicolumn{2}{|c|}{ Prostate (physician $1^{*}$ ) } & \multicolumn{2}{|c|}{ Prostate (physician 2*) } \\
\hline & Preference & Degree & Preference & Degree & Preference & Degree \\
\hline Physician generated plan & 3 & all slightly & 2 & 1 slightly, 1 significantly & 5 & 3 slightly, 2 significantly \\
\hline Clinical plan & 6 & 5 slightly, 1 significantly & 6 & 4 slightly, 2 significantly & 3 & 2 slightly, 1 significantly \\
\hline No preference & 3 & - & 2 & - & 2 & - \\
\hline Total & 12 & - & 10 & - & 10 & - \\
\hline
\end{tabular}

order to achieve the average of the presented plans. Prostate plans were additionally rated by a non-planning involved physician who voted in favor of physician plans.

\section{Physicians' experiences}

On a more qualitative basis, recorded physicians' statements during the sliding sessions, such as: "it is interesting how this dose increases while the other one decreases" or "that was a good deal" demonstrated the physicians appreciating having the control over the dose. Experiencing dosimetric trade-offs improved their comprehension of the planning situations.

\section{Planning efficiency \\ Planners' time}

The first planning step, the beam selection, is identical for both planning approaches (Fig. 3). In the second step physician driven planning reduces planners' time through the utilization of pre-defined optimization templates, which can save up to $\Delta t_{2}=10 \mathrm{~min}$, the approximate average time it takes to decide on objectives and constraints and add them to the formulation. For step 3, in clinical planning, the required number of iterations $\mathrm{N}$ until a plan is approved (see above) is determined to be $N=1$ for prostate planning, as it is a standardized procedure and plans are mostly accepted at the first review. For brain tumor cases $\mathrm{N}$ varies between one, two, and occasionally three. We assume an average of $N=1.5$ per plan. Physician driven planning eliminates planners' navigation completely which corresponds to a time of approx. $10 \mathrm{~min}$ in simpler cases (see e.g. [1]) and up to $30 \mathrm{~min}$ in complex cases. Considering the factors of 1.0 and 1.5 (iterations), planner time $\Delta \mathrm{t}_{3, \mathrm{pl}}$ decreases by 10 and $45 \mathrm{~min}$ for prostate (Fig. 3a) and brain cases (Fig. 3b), respectively.

\section{Physician times}

Clinical physician times to coordinate and communicate with planners are on average $2 \mathrm{~min}$ for prostate and 5 min for brain cases. Plan review and approval take approximately $5 \mathrm{~min}$ for prostate and $10 \mathrm{~min}$ for brain cases (complexity comparable to lung tumors, average times published by [23]). Taking the number of iterations into account, physician times were estimated by $7 \mathrm{~min}$ for prostate $\left(\mathrm{N}=1 ; \mathrm{t}_{\mathrm{ph}}(\mathrm{CP})=\mathrm{t}_{3, \mathrm{ph}}(\mathrm{CP}) \cong 2+\right.$ $5 \mathrm{~min})$ and $20-25 \mathrm{~min}$ for brain cases $(\mathrm{N}=1.5$; $\left.\mathrm{t}_{\mathrm{ph}}(\mathrm{CP})=\mathrm{t}_{3, \mathrm{ph}}(\mathrm{CP}) \cong 1.5 \times 15 \mathrm{~min}\right)$.

Average physician navigation times were $10 \mathrm{~min}$ for prostate and $16 \mathrm{~min}$ for brain tumor. Distinguishing average times by the first and second half of navigated plans, times decreased from 13 to 7 min (averages over 5 plans) and 17 to $15 \mathrm{~min}$ (averages over 6 plans) for prostate and brain tumors, respectively.

The achieved physician time savings between both approaches were calculated by subtracting the average navigation time (after more training) from the clinical involvement times, which results in no time difference for prostate $\left(t_{\mathrm{ph}}(\mathrm{PP})=\mathrm{t}_{3, \mathrm{ph}}(\mathrm{PP}) \cong 7 \mathrm{~min}\right.$ ) (Fig. 3a) and between 5 and $10 \mathrm{~min}$ for brain tumor cases $\left(t_{\mathrm{ph}}(\mathrm{PP})=\mathrm{t}_{3, \mathrm{ph}}(\mathrm{PP}) \cong 15 \mathrm{~min}\right)$ (Fig. $3 \mathrm{~b}$ ), corresponding to a reduction of $30 \%$ of the involved time.

\section{Discussion}

We presented a planning approach with physician navigation as a defining part of the process. While making planning more patient independent by creating standardized databases, our approach makes it more individualized for each patient by not following the same standard prescription for everyone but finding the best trade-offs for every individual. This study sought to determine if standardized MCO templates plus physician navigation of the resulting Pareto surfaces could offer a viable alternative to the standard planning process, where tradeoffs are explored by the treatment planners and the physicians are only involved in the final YES/ NO decision.

Our retrospective planning study is subject to limitations given by inherent differences between clinical and physician driven planning. Although clinical plans were generated by different dosimetrists, they were regarded as comparable. Different qualities within plans may have resulted due to individual planning strategies and personal preferences. The utilization of different TPS software versions may have led to a slight advantage in the navigation process for physicians. As dosimetrists are well trained to the clinical software version, and as the intention of the study was not to compare minor but 
rather fundamental dose differences, this difference is assumed not to have an impact on the study results (for details on dosimetric differences between the two software versions see [21]). Further limitations are found in the restricted number and variety of cases and of participating planning physicians (one for each case).

As the study was performed retrospectively, the exact clinical planning times were not recorded, which prohibited a plan by plan comparison of required times for each step of the procedure. Instead we reverted to clinical experiences of staff members and estimated average times. Commonly, physician times are scarce due to their clinical schedules, and the most expensive. This motivates the aim to shorten physician involvement by utilizing their time as best as possible. While for prostate cases, required times to navigate were comparable to the times to review and approve plans, physician driven planning achieved sizeable time reductions for brain tumors. Compared to prostate planning, these more complex cases are usually subject to more dosimetric conflicts and require more communication. Figure 5 presents a brain tumor case for which the physician prioritized better target coverage over the hard constraint on the brainstem. While dosimetrists usually respect formulated hard constraints, physicians will not always rigidly adhere to their standard prescriptions when compromises are required. Not only in cases of incompatible goals but also when all constraints are achievable simultaneously, physician background knowledge is important in order to decide how best to distribute or escalate the dose.

Contrary to possible expectations, the blinded comparison showed a slight preference (not statistically significant) for the clinical plans for both physicians. In our view, this reflects the fact that the treatment planners 1 ) are experienced and 2) have effective working relationships with the physicians such that they can achieve plans deemed desirable by both physicians. In light of these ideas, we find it encouraging from the perspectives of increased throughput and increased physician involvement that the plan quality of both planner and physician generated plans are on par. Picking a preference between two plan options was difficult. To examine whether the preference decisions were stable, prostate plans were rated by a second non-planning involved physician. While the planning physician voted 2:6 for the physician vs. the clinical plan (Table 2), the second doctor voted $5: 3$, favoring the physician plans. Both physicians chose equally good twice but on different cases.

The choice of preferences demonstrated the interobserver variability [24] of opinions on plan quality. Generally, plan preferences are considered to be caused by personal clinical experiences, e.g. having recently experienced certain patient side-effects might lead to a different point of view. Having a second physician review the cases highlighted the result from the initial physician selections that the two plans for each case are both acceptable plans.

Due to the ongoing learning process of physicians throughout the study, a final conclusion on physicians' dose preferences might be too early and would require a planning study with more physicians at a later stage of MCO-training. This feasibility study was performed with minimal trained physicians to demonstrate that physicians can do plan navigation. Further improvements of plan qualities are expected by improving optimization formulations, e.g. by adapting objective functions and structures to physicians' needs instead of dictating them.

Generally, the choice of optimization parameters is crucial. Translating physicians' desires for each organ by one slider is challenging and not always possible, given that judging the dose distribution on even a single organ is a multi-dimensional task [10]. In general it is difficult to steer three dimensional dose distributions by using one criterion, e.g. trying to get rid of a hot spot at a certain position might reduce dose of that structure at a different location simultaneously. The development of objective functions that allow for a more accurate and specific steering and control of the doses could improve planning in the future.

Our study provided insight into the planning process to physicians with which they are often not familiar. Involving physicians is not only feasible but can also be regarded as a gain of knowledge on more levels. Knowing the expense of a certain dosimetric goal like a homogeneous target dose distribution might change the physicians' point of view or expectations to a plan [25]. Even if clinical planning and navigation is not done by the physicians it is a gain for the physician to understand the process, and experience the trade-offs also for comprehending difficulties planners sometimes face.

MCO-treatment planning could also serve as a tool for educational purposes and in clinicians' rounds. Being aware of realizable dose distributions, physicians could better specify the prescriptions and clinical goals of each individual case [26]. Clearer formulated prescriptions will shorten the "back and forth" process between planner and physician and thus make the planning process more efficient.

The suggested procedure may serve as a basis for a discussion to refine optimal planning workflow. Obviously the planning cannot be done by physicians only, as the whole plan creation would be too time consuming for an efficient clinical workflow and best utilization of clinician time. Here physicians were provided with the prepared database, including prior required work steps such as beam angle selections, and physician involvement consisted of the final plan navigation and 
generation. The whole planning process is and will stay an interactive process of a mix of professions. Our study did not demonstrate the general superiority of this approach, but indicated that deviation from the standard procedure has the potential to improve planning efficiency, and possibly quality (physician navigation skills improved over time). Finding the "best" approach to this interactive process of different professions, in terms of efficiency and resulting quality, still requires further investigations.

It could be promising to combine MCO with knowledgebased planning [7-9, 27, 28]. While automated knowledgebased planning can generate good plans that are sometimes even preferable to planner-generated IMRT plans, physicians still need to apply clinical judgment to make the best treatment decision for each individual patient. In our experience, the best way to accomplish this is through the use of MCO - except for the most trivial cases.

Knowledge based planning and $\mathrm{MCO}$ are both needed and they are synergistic: the knowledge-based system could provide the templates and beam orientations, as well as the starting point for the interactive navigation. The physician would then be involved in making relatively minor adjustments using only a small number of sliders/trade-offs, without being overwhelmed by too many options.

We believe that planning systems of the future will be both knowledge-based and MCO-based.

\section{Conclusion}

We demonstrated the feasibility of physician driven planning in MCO treatment planning by Pareto surface navigation on template based optimized plans. The plan quality of physician generated plans was comparable to the clinical plans with differences being observed due to focusing on different clinical goals. This workflow always reduces the required planner time while the reduction in physician time is case dependent and potentially greater for more complex cases. Although the evidence for superior plan quality was not proven within this study, and is certainly also case and patient dependent, it demonstrated that increasing physician involvement and at an earlier stage into the planning process is feasible and has the potential to improve departmental efficiency.

\section{Endnotes}

${ }^{1}$ planning personnel varies between countries and clinics; in the following "planner" refers to both professions.

\section{Abbreviations}

CTV: Clinical target volume; GTV: Gross tumor volume; IMRT: Intensity modulated radiotherapy; MCO: Multicriteria optimization; MGH: Massachusetts General Hospital; MLC: Multileaf collimator; OAR: Organ at risk; PTV: Planning target volume; TPS: Treatment planning system

\section{Acknowledgements}

This study was supported in part by the Federal Share of program income earned by Massachusetts General Hospital on C06-CA059267. Proton Therapy Research and Treatment Center. Support was also provided by RaySearch Laboratories AB, Stockholm, Sweden. Further support was obtained from the DFG cluster of excellence: Munich-Centre for Advanced Photonics and the TUM Graduate School. The authors thank Anthony Zietman for his participation in this project.

\section{Funding}

The study was supported in part by a research grant from RaySearch Laboratories AB, Stockholm, Sweden (grants received by TB and DC). Further grants were obtained from German Research Foundation (DFG) during the conduct of the study (grants received by BM).

\section{Availability of data and materials}

The datasets used and/or analyzed during the current study available from the corresponding author on reasonable request.

\section{Authors' contributions}

Study design: BM, TB, DC. Data collection, analysis and development of methods: BM, DC. Planning: BM, HS, JE. Interpretation and discussion of data: all authors. Manuscript writing: all authors. Manuscript review and final approval: all authors.

\section{Ethics approval and consent to participate}

Not applicable

\section{Consent for publication}

Not applicable

\section{Competing interests}

The study was supported in part by a research grant from RaySearch Laboratories AB, Stockholm, Sweden (grants received by TB and DC). HS reports being an editor for the IJROBP.

\section{Publisher's Note}

Springer Nature remains neutral with regard to jurisdictional claims in published maps and institutional affiliations.

\section{Author details}

${ }^{1}$ Department of Radiation Oncology, Massachusetts General Hospital, Harvard Medical School, Boston, MA, USA. ${ }^{2}$ Department of Radiation Oncology, Klinikum rechts der Isar, Technical University of Munich, Ismaninger Straße 22, 81675 Munich, Germany. ${ }^{3}$ Department of Physics, Technical University of Munich, Munich, Germany.

Received: 1 February 2017 Accepted: 20 October 2017

Published online: 06 November 2017

\section{References}

1. Wala J, Craft D, Paly J, et al. Maximizing dosimetric benefits of IMRT in the treatment of localized prostate cancer through multicriteria optimization planning. Med Dosim. 2013;38:298-303.

2. Lahanas M, Schreibmann E, Baltas D. Constrained free gradient-based optimization algorithms for multiobjective inverse planning in intensity modulated radiotherapy. Phys Med Biol. 2003;48:2843-71.

3. Haas OCL. Radiotherapy treatment planning: new system approaches, advances in industrial control monograph. London: Springer Verlag; 1999.

4. Voet PWJ, Dirkx MLP, Breedveld S, et al. Toward fully automated Multicriterial plan generation: a prospective clinical study. Int J Radiat Oncol Biol Phys. 2013;85:866-72.

5. Xhaferllari I, Wong E, Bzdusek K, et al. Automated IMRT planning with regional optimization using planning scripts. J Appl Clin Med Phys. 2013;14: 176-91.

6. Jolly D, Alahakone D, Meyer J. A RapidArc planning strategy for prostate with simultaneous integrated boost. J Appl Clin Med Phys. 2011;12:35-49.

7. McNutt T, Wu B, Moore J, et al. Automated treatment planning using a database of prior patient treatment plans [abstract]. Med Phys. 2012;39:4008. 
8. Moore J, Yang W, Kimberly ET, et al. Clinical deployment of automatic treatment planning for pancreas SBRT patients [abstract]. Int J Radiat Oncol Biol Phys. 2013;87(Suppl):S136-7.

9. Moore JA, Evans K, Yang W, et al. Automatic treatment planning implementation using a database of previously treated patients. J Phys Conf Ser. 2014;489:012054.

10. Craft DL, Hong TS, Shih HA, et al. Improved planning time and plan quality through multicriteria optimization for intensity-modulated radiotherapy. Int J Radiat Oncol Biol Phys. 2012;82:e83-90.

11. Kierkels RGJ, Visser R, Bijl HP. Multicriteria optimization enables less experienced planners to efficiently produce high quality treatment plans in head and neck cancer radiotherapy. Radiat Oncol. 2015;87(10). doi:10.1186/ s13014-015-0385-9.

12. Yu Y, Zhang JB, Cheng G, et al. Multi-objective optimization in radiotherapy: applications to stereotactic radiosurgery and prostate brachytherapy. Artif Intell Med. 2000;19(1):39-51.

13. Cotrutz C, Lahanas M, Kappas K, et al. A multiobjective gradient based dose optimization algorithm for external beam conformal radiotherapy. Phys Med Biol. 2001;46(8):2161-75.

14. Haas OCL, Burnham KJ, Mills JA. Optimization of beam orientation in radiotherapy using planar geometry. Phys Med Biol. 1998;43(8):2179-93.

15. Craft D, Halabi T, Bortfeld T. Exploration of tradeoffs in intensity-modulated radiotherapy. Phys Med Biol. 2005;50:5857-68.

16. Thieke C, Bortfeld T, Niemierko A, et al. Multicriteria optimization in inverse radiotherapy planning. Radiother Oncol. 2003;68(Suppl. 1):44. (Abstract), pre-meeting Workshop on Optimization of IMRT, 7th Biennial ESTRO Meeting on Physics and Radiation Technology for Clinical Radiotherapy, Geneva, Switzerland

17. Thieke C, Küfer K-H, Monz M, et al. A new concept for interactive radiotherapy planning with multicriteria optimization: first clinical evaluation. Radiother Oncol. 2007:85(2):292-8.

18. Küfer K-H, Hamacher HW and Bortfeld TR. A multicriteria optimization approach for inverse radiotherapy planning. Proc. XIIIth ICCR (Heidelberg), ed. TR Bortfeld and W Schlegel. 2000;9-26.

19. Breedveld S, Storchi PRM, Heijmen BJM. The equivalence of multi-criteria methods for radiotherapy plan optimization. Phys Med Biol. 2009;54(23): 7199-209.

20. Fredriksson A, Bokrantz R. Deliverable navigation for multicriteria IMRT treatment planning by combining shared and individual apertures. Phys Med Biol. 2013;58:7683-97.

21. Kyroudi A, Petersson K, Ghandour S, et al. Discrepancies between selected Pareto optimal plans and final deliverable plans in radiotherapy multicriteria optimization. Radiother Oncol. 2016;120(2):346-8.

22. Bokrantz R. Multicriteria optimization for managing tradeoffs in radiation therapy treatment planning. PhD thesis 2013.

23. Kamran SC, Müller BS, Paetzold P, et al. Multi-criteria optimization achieves superior normal tissue sparing in a planning study of intensity-modulated radiation therapy for RTOG 1308-eligible non-small cell lung cancer patients. Radiother Oncol. 2016;1 18(3):515-20.

24. Vinod SK, Jameson MG, Min M, et al. Uncertainties in volume delineation in radiation oncology: a systematic review and recommendations for future studies. Radiother Oncol. 2016;121:169-79.

25. Craft D, Khan F, Young M, et al. The price of target dose uniformity. Int J Radiat Oncol Biol Phys. 2016;96(4):913-4.

26. Otto K. Real-time interactive treatment planning. Phys Med Biol. 2014;59: 4845-59.

27. Yuan $L$, Wu QJ, Sheng Y, et al. Local multi-criteria optimization for clinical tradeoff decision guidance in RT planning. Med Phys. 2015;42(6):3459. (abstract)

28. Zarepisheh A, Long T, Li N, et al. A DVH-guided IMRT optimization algorithm for automatic treatment planning and adaptive radiotherapy replanning. Med Phys. 2014;41:061711.

29. Niemierko A. A generalized concept of equivalent uniform dose (EUD). Med Phys. 1999;26:1100. (abstract)

\section{Submit your next manuscript to BioMed Central and we will help you at every step:}

- We accept pre-submission inquiries

- Our selector tool helps you to find the most relevant journal

- We provide round the clock customer support

- Convenient online submission

- Thorough peer review

- Inclusion in PubMed and all major indexing services

- Maximum visibility for your research

Submit your manuscript at www.biomedcentral.com/submit
C) Biomed Central 